\title{
Civilisations
}

Revue internationale d'anthropologie et de sciences

humaines

40-1 | 1991

L'Asie et le Pacifique

\section{Le Nouveau Monde du Pacifique}

Jacques Groothaert

URL : http://journals.openedition.org/civilisations/1676

DOI : 10.4000/civilisations. 1676

ISSN : 2032-0442

\section{Éditeur}

Institut de sociologie de l'Université Libre de Bruxelles

\section{Édition imprimée}

Date de publication : 1 janvier 1992

Pagination : 25-41

ISBN : 2-87263-063-5

ISSN : 0009-8140

\section{Référence électronique}

Jacques Groothaert, «Le Nouveau Monde du Pacifique », Civilisations [En ligne], 40-1 | 1991, mis en ligne le 07 juillet 2009, consulté le 19 avril 2019. URL : http://journals.openedition.org/ civilisations/1676; DOI : 10.4000/civilisations.1676 


\section{LE NOUVEAU MONDE DU PACIFIQUE}

\section{Jacques GROOTHAERT}

L'évolution dramatique de l'Europe centrale et orientale est bien entendu au coeur de toutes les préoccupations. La présence, au récent Forum Economique mondial de Davos (février 1990) d'un grand nombre de dirigeants et de responsables de ces pays, a suscité un intérêt particulier. Mais on y a entendu aussi un avertissement de poids, dans le discours remarquable qu'a prononcé le Premier Ministre de Singapour Lee Kwan Yew, qui préside depuis trente ans aux destinées et au prodigieux succès de ce petit pays, situé au centre nerveux d'une région sud-est asiatique en pleine évolution.

\section{Il disait :}

"Le chemin pourra être plus long et plus difficile que ne le réalisent les Européens de l'Est. La Chine a découvert qu'il n'est pas si aisé de rejeter les effets de 40 ans de marxismeléninisme...

Il serait triste de voir l'Europe occidentale absorbée par la tâche de reconstruction des économies de l'Europe de l'Est, au point de se révéler incapable de concurrencer le Japon et les Etats-Unis dans les économies en pleine croissance de l'Asie et du Pacifique. Pour les années à venir, l'Europe de l'Est ne pourra se comparer au dynamisme des pays du "Pacific Rim" qui jouent un rôle décisif dans le progrès technologique.

Les Européens de l'Ouest ne peuvent se permettre, même temporairement, d'être absents dans cette partie du monde. Ils trouveront de bons partenaires dans les pays nouvellement industrialisés (les 4 "dragons") et ceux de l'ASEAN, qui tous souhaitent un ordre économique en Asie qui ne soit pas dominé par le Japon" ! 
Le "monde du Pacifique" s'étend bien au-delà de ce sud-est asiatique en spectaculaire expansion. Il inclut, bien sûr, l'Australie, et aussi le versant Pacifique du Canada, des EtatsUnis, de l'Amérique latine. L'immense Océan Pacifique, vaste comme deux fois l'Atlantique, est en réalité une "nouvelle Méditerranée", dont les rivages sont habités par près de six habitants de la planète sur dix, créant une communauté de fait vers laquelle s'opère un glissement du centre de gravité économique mondial.

Devant l'émergence de cette réalité, voici que de toutes parts on exhume les prédictions de Théodore Roosevelt proclamant, au début de ce siècle, que :

"L'histoire de l'humanité a débuté par une ère méditerranéenne, elle a continué par une période atlantique et elle entre actuellement dans une phase du Pacifique",

et même de Karl Marx (qui ne s'est donc pas toujours trompé), écrivant dans la Neue Rheinische Zeitung du 11 février 1858 (!):

"Le Pacifique jouera le rôle joué maintenant par l'ocćan Atlantique et au moyen-âge par la Méditerranée : le rôle de la grande route maritime du trafic mondial".

En les commentant, dans un remarquable article dans $L e$ Monde du 28 avril 1984, André Fontaine observait que :

"Les mers ont engendré au cours des âges des courants d'échanges commerciaux et intellectuels, des alliances, à la limite des symbioses, autrement solides, de maniere générale, que les unions continentales, dont la brièveté est la décourageante règle.

(...) Le bassin du Pacifique a mis plus longtemps que les autres, du fait de son éloignement de l'Europe... et aussi de son étendue. Grâce au prodigieux raccourcissement des distances c'est chose faite aujourd'hui. 
Il importe de considérer le rôle des principaux acteurs de ce grand jeu du Pacifique, tant sur le plan économique qu'au niveau géo-politique et stratégique, et leur interaction.

Leur "manifest destiny" a progressivement porté les Américains vers l'Ouest, d'abord de leur propre continent, puis au-delà de leur façade orientale qu'ils ont tardivement peuplée : en 1845, il y avait 700 Américains en Californie...

Dès la moitié du 19e siècle, cependant, l'escadre du commodore Perry forçait - avec les conséquences que l'on connaît - l'ouverture au commerce mondial du Japon si longtemps isolé. Les Etats-Unis acquéraient Hawaï, les Philippines, voyaient le Pacifique se transformer en une sorte de "lac américain". Leur expansion devait inévitablement se heurter à l'ambition du rival japonais qui lui aussi, en était venu à voir sa destinée manifeste dans la création d'une vaste zone de domination économique. De Pearl Harbour à Hiroshima, aux guerres de Corée et du Vietnam, l'Amérique se verra durablement engagée dans des conflits dont les derniers furent motivés par sa volonté d'endiguer la menace de l'hégémonie communiste dans cette partie du globe. Cela l'a conduite à sceller avec le Japon vaincu, et rapidement reconstruit, une alliance militaire d'une importance demeurée essentielle face aux incertitudes stratégiques de l'avenir. Les grandes doctrines diplomatiques américaines ont été énoncées à la suite des défis lancés en Asie.

On a pu voir, au fil des années récentes, se dessiner une orientation croissante de l'intérêt américain vers la zone du Pacifique. A une époque où l'Europe semblait en proie à une Eurosclérose bientôt, ô combien, démentie, s'élevèrent des voix en faveur d'une réorientation politique et économique vers ce 
"Near West" qu'était devenu pour certains Américains l'Extrême-Orient.

Pour les auteurs de "The Third Century : America's Resurgence in the Asian Era", Joel Kotkin et Yoriko Kishimoto, "c'est en Asie, et non en Europe, qu'est l'avenir".

Un Japonais influent, Kenichi Ohmae, parlait de la "Californisation du monde libre", et pour Hélène Carrère d'Encausse

"La façade atlantique des Etats-Unis avait été celle de l'ère industrielle. Depuis plus de 20 ans déjà, la façade pacifique est devenue celle de l'ère post-industrielle : l'univers de la technologie de pointe et de l'informatique.

Les Etats-Unis sont donc occupés à quitter le monde auquel nous appartenons pour basculer vers ce Pacifique en pleine expansion".

Par ailleurs, la présence des Asiatiques aux Etats-Unis, en particulier sur la côte Ouest, ne cesse de s'affirmer, dans l'industrie et dans les universités en particulier, où ils s'imposent par leur plus haut degré d'éducation et de formation ${ }^{1}$, leur ardeur au travail, les valeurs éthiques confucianistes qui marquent la plupart d'entre eux. A la fin de ce siècle, les Américains d'origine asiatique pourraient être dix millions.

Mais une certaine euphorie "Pacifique" a rapidement fait place à un revirement spectaculaire, provoqué par l'extraordinaire progrès de l'économie japonaise, les investissements nippons aux Etats-Unis (l'achat par les Japonais du Rockefeller Center a un effet traumatisant), leur puissance financière. De récents sondages d'opinion font apparaître que désormais, du fait de la détente, une majorité d'Américains se montrent plus préoccupés par la menace 
industrielle et commerciale nippone que par une puissance soviétique en déclin.

Une école dite "révisionniste" alerte l'opinion américaine à propos de ce nouveau péril, conjugué avec la perception du recul industriel et technologique des Etats-Unis. Clyde Prestowitz publie "Trading Places" qui veut démontrer "comment les Etats-Unis ont permis au Japon de les détrôner". Les ouvrages de Fallows, de van Wolferen sur "l'énigme du pouvoir japonais" sont analysés avec passion. On prône une attitude dure, on réclame un protectionnisme anti-nippon.

Néanmoins, la compréhensible inquiétude américaine est mitigée par la conscience de la nécessité géo-politique d'un partenariat à travers l'océan Pacifique et certains, une fois retombées les prédictions apocalyptiques, entrevoient par contre, à terme, une forme de symbiose américano-japonaise, une double hégémonie ou condominium sur l'ensemble de la zone du Pacifique.

Le secrétaire d'état James Baker disait, devant l'Asia Society à New York le 26 juin 1989 :

"Les uniques capacites politiques, économiques et militaires des Etats-Unis assurent les bases d'une zone Pacifique prospère et sûre. Celles-ci se verront renforcées par des partenariats régionaux et nul n'est plus important que notre alliance avec le Japon. Nous vivons dans une interconnection étroite... Le temps est venu pour le Japon de traduire ses succès domestiques et régionaux par l'occupation d'un rôle international impliquant des responsabilités accrues".

Beaucoup de dirigeants américains demeurent convaincus que malgré la concurrence, les rivalités et les conflits commerciaux, les Etats-Unis et le Japon sont des alliés naturels: 
ils n'ont pas vraiment le choix. Très révélateur à cet égard est l'article publié dans The Times du 28 février 1990 par Zbigniew Brzezinski $Z$ b. qui va jusqu'à plaider pour un "arrangement pan- Pacifique" qu'il souhaite appeler "Amerippon" !

Personne aujourd'hui ne nie que le Japon a atteint, et audelà, par son expansion économique, les buts qu'il s'était assignés en déclenchant les hostilités il y a cinquante ans. La "zone de co-prospérité" qu'il avait voulu créer et dominer par les armes, est devenue à présent une vaste région de complémentarité économique, de croissante intégration industrielle. Les investissements japonais sont omni-présents dans tout le Sud-Est asiatique, où ils ont organisé de facto une vaste division du travail et financent l'infrastructure industrielle des nouveaux pays en voie d'industrialisation.

La réussite technologique du Japon est évidente. Il devient, a-t-on pu dire, exportateur des matières premières du futur, celles à forte teneur en matière grise, et les produits nouveaux issus de ces technologies deviendront aussi nécessaires que l'est actuellement le pétrole. "La révolution électronique est la première révolution technologique qui ne sera pas née en Europe, mais dans la région du Pacifique".

Wisse Dekker, ancien président de Philips, estime que le plan stratégique japonais prévoit la production en 1992 de l'ordinateur de la 5ème génération basé sur l'intelligence artificielle, un projet selon lui comparable à l'envoi d'un homme sur la lune, mais avec des conséquences sociales, économiques et politiques plus grandes. Cela représentera une rampe de lancement pour le rôle dominant que l'Extrême-Orient et le Japon en particulier, jouera grâce à son avance technologique ${ }^{2}$. 
Les Japonais, a-t-on pu dire, sont en fait "les Occidentaux de l'Asie". Ils se situent, à bien des égards à la périphérie du continent asiatique ${ }^{3}$.

Mais la réalité économique pousse inévitablement le Japon à s'affirmer comme le centre et le moteur d'un vaste regroupement, d'une zone de complémentarité, où il est devenu le dispensateur $n^{\circ} 1$ d'aide au développement, le premier investisseur et le premier partenaire. Les entreprises japonaises font de plus en plus transférer leur production vers les pays de la zone. La majorité des exportations des pays de l'ASEAN est le fait de filiales japonaises. Les "dragons" de l'Est asiatique n'ont d'autre choix que d'être solidaires de leur puissant voisin.

Ceux que l'on a appelés les "quatre dragons" (Corée du Sud, Taïwan, Hong Kong et Singapour) tiennent une place de plus en plus importante dans la production industrielle et dans les échanges commerciaux mondiaux. Conjointement avec les six pays de l'ASEAN (Malaisie - Indonésie - Thaïlande Singapour - Philippines - Brunei) - où la Thaïlande a l'ambition de devenir le "cinquième dragon" à brève échéance, ils constituent une zone de croissance et de dynamisme remarquable. La Corée, en particulier, affirme ses ambitions, se pose dans certains domaines en rival économique du Japon, étend ses propres investissements hors de ses frontières, montre un intérêt particulier pour l'Europe Centrale et Orientale et effectue en direction de l'U.R.S.S. un rapprochement bien accueilli à Moscou.

D'autre part, l'Australie se détournant quelque peu de ses racines européennes, a clairement pris conscience du fait que ses intérêts désormais ne la lient plus guère au Commonwealth, mais bien à ses proches voisins asiatiques. On parle de la 
"ferveur pro-asiatique" des Australiens, qui privilégient la géographie par rapport à l'histoire.

Reste la Chine. Une fois encore, elle est en passe de décevoir les espérances qu'avait pu faire naître son récent effort d'ouverture et de modernisation. Elle semble se montrer plus que jamais réticente à jouer un rôle effectif de puissance du Pacifique - à l'exception de l'intérêt qu'elle montre pour les ressources pétrolières au large de ses côtes - d'où ses conflits avec le Vietnam pour le contrôle de l'archipel des Spratley.

Mais on paraît voir s'estomper la perspective de la création d'une zone de prospérité dans les provinces côtières chinoises où, depuis la création de "zones économiques spéciales" certains voyaient s'ébaucher plusieurs "mini Hong Kong", formant un important chapelet de pôles économiques tournés à la fois vers la Chine centrale et vers l'extérieur.

Cette évolution, souhaitée par le disgrâcié Zhao Ziyang, est en butte aux jalousies des autres provinces et suscite la crainte d'un pouvoir central soucieux de ne pas voir lui échapper des régions que pourrait tenter l'autonomie.

On voit aussi Pékin - au mépris de ses intérêts économiques évidents et de ses perspectives d'avenir - durcir son attitude envers Hong Kong, provoquant ainsi l'exode des cerveaux au risque de ruiner un potentiel qui devrait pourtant lui être indispensable. Ceux qui craignent la permanence des vieuxx démons chinois n'ont sans doute pas tort.

Dans la nouvelle phase de l'évolution mondiale qui se dessine, les Etats-Unis vont difficilement résister à la tentation 
de réduire leur présence militaire en Extrême-Orient, mais, on peut l'espérer, sans aller trop loin dans un désengagement qui pourrait, à la fois, se révéler dangereusement prématuré, encourager une instabilité latente du fait de conflits interrégionaux toujours prêts à s'allumer, et laisser par trop le champ libre à une présence militaire japonaise que personne, dans la région, ne veut voir s'amplifier.

Le Japon ne consacre "qu'un pourcent" de son PNB à la défense. Mais ce PNB est à ce point gigantesque, qu'il fait du Japon la troisième puissance militaire mondiale en termes de dépenses militaires ! Tokyo demeure sceptique face à la réalité de la politique de détente de Moscou, où l'on ne se prive pas, au demeurant, de mettre en garde contre le revanchisme et le militarisme nippon!

On l'oublie parfois : la Russie était présente sur les rivages du Pacifique cent cinquante ans avant les Américains... Dès le 17e siècle, la pénétration des immenses territoires au-delà de l'Oural s'était faite avec opiniâtreté et, vu les énormes difficultés dues au climat et aux distances, en un laps de temps relativement court.

Engagés dans le fructueux commerce des fourrures, et en particulier la chasse à la loutre marine, les pionniers russes avaient atteint et occupé l'Alaska et établi des postes avancés sur la côte californienne. Un navire russe se présenta, au début du 19e siècle dans le petit port - encore espagnol - qui allait devenir, quelques années plus tard, San Francisco ! Combien le cours de l'histoire eût pu être différent si cette éphémère présence russe s'était consolidée! 
On sait qu'il n'en fut rien : trop éloignés de leurs bases européennes, les colonisateurs russes renoncèrent bien vite, et l'Alaska fut cédé, en 1867, aux Etats-Unis, pour une somme dérisoire...

En 1905, la victoire du Japon sur l'Empire russe, marquant l'irrésistible progression nipponne, semblait sonner le glas des ambitions russes dans l'Extrême-Orient. Pourtant, celles-ci se sont réaffirmées, avec l'expression non-équivoque d'une volonté de puissance, à l'issue de la seconde guerre mondiale.

Aujourd'hui, l'U.R.S.S. est présente dans le Pacifique et avait commencé à y renforcer ses positions économiques et stratégiques. Son effort dans le domaine naval s'est révélé d'une grande ampleur. La flotte soviétique du Pacifique compte quelque 850 navires, dont deux porte-avions et 25 sous-marins nucléaires. A l'heure actuelle, elle apparait en voie de réduction assez marquée.

L'U.R.S.S. pourrait, entend-on dire, fortement diminuer sa présence dans les bases de Danang et de Cam Ranh, au Vietnam, face aux bases américaines de Clark et Subic aux Philippines (elles-mêmes au statut incertain). Elle en a acquis d'autres sur les côtes de la Corée du Nord. Gardant sous son contrôle les îles Kouriles - que le Japon souhaite vainement récupérer - la puissance navale soviétique se voulait en mesure de contrôler, dans l'immense arc de cercle qui va du détroit de Behring à celui de Malacca, certaines des voies maritimes commerciales les plus importantes du monde. Par ailleurs, tout au long de la frontière chinoise, l'U.R.S.S. avait jusqu'il y a peu concentré des forces impressionnantes. Ces déploiements étaient à la mesure de ses ambitions de grande puissance 
omniprésente. Là aussi, la politique de Gorbachev va dans le sens d'une nette réduction.

Ayant affirmé sa force et sa vocation, l'Union soviétique apparaît disposée au dialogue. La période de tension extrême avec la Chine est dépassée : un conflit militaire semble désormais fort improbable, et les relations commerciales sinorusses ont pris récemment un nouvel essor (les échanges pourraient atteindre à la fin de cette décennie un montant de 6 milliards de dollars).

Le Japon, toujours inquiet devant la présence militaire soviétique qu'il perçoit comme une réelle menace, s'est donné les moyens de contrôler les détroits stratégiques qui sont le passage obligé des navires russes quittant leurs bases extrêmeorientales pour se diriger vers les mers du Sud. L'ex-Premier Ministre Nakasone décrivait, il n'y a guère, le Japon comme un "porte-avions insubmersible". Tokyo ne demeure pas, pour autant, insensible aux offres de coopération industrielle - dont certaines ont déjà donné lieu à des réalisations - pour le développement des vastes ressources sibériennes, où la technologie nipponne faciliterait la mise en valeur du versant Pacifique de l'U.R.S.S.

Cette dernière a approuvé le principe d'une série de jointventures avec les Japonais, dont les "trading houses" montrent de l'intérêt pour les perspectives de coopération industrielle et commerciale avec la région sibérienne (bois, pétrole, gaz, charbon), au moment où du fait de la chute des prix du pétrole, l'Union soviétique a grand besoin de nouvelles ressources en devises.

Un statut de zone économique spéciale à Nakhodka, dans la région maritime extrême-orientale, prend forme, et on espère y 
attirer des investissements non seulement japonais, mais américains.

Toutefois, du côté japonais, la méfiance persiste : Tokyo ne semble pas trop croire en la réussite de la perestroïka, et demeure farouchement opposé à un rapprochement tant que l'U.R.S.S. n'aura pas accepté de revoir le statut des quatres îles de l'archipel des Kouriles dont le Japon persiste à revendiquer le retour à la mère-patrie...

Le discours prononcé le 28 juillet 1986 par Mikhail Gorbachev à Vladivostok avait révélé l'importance accordée par le gouvernement soviétique à l'affirmation de son intérêt pour l'ensemble de la région du Pacifique. Il rappelait que "la plus grande partie de notre territoire est à l'est de l'Oural" (en 1966, de Gaulle avait découvert que l'Europe ne va pas de l'Atlantique à l'Oural, et parlait, à son retour d'une viste à Novosibirsk, de "l'Europe d'un bout à l'autre"...!).

Gorbachev voit en Vladivostok "un futur grand centre international, foyer de commerce et de culture, une fenêtre largement ouverte sur l'Orient...".

Evgeny Primakov, qui a dirigé l'Institut des relations économiques internationales, rappelait que "l'Extrême-Orient est part intégrante de notre économie nationale", et doit être intégré de façon autonome dans une division internationale du travail dans le Pacifique.

L'U.R.S.S. soutient l'idée d'une zone dénucléarisée dans le Pacifique. Elle se dit prête à participer à des négociations sur la réduction généralisée de l'activité navale dans la région. Elle établit des relations étroites avec les nouveaux Etats de 
Vanuatu et Kiribati et conclut des accords de pêche qui mettent en cause le monopole américain.

Cela dit, pour nombre d'observateurs, le véritable objectif de toutes les initiatives et propositions soviétiques serait avant tout la Chine, dont le développement et l'orientation demeurent la préoccupation essentielle de l'U.R.S.S.

Dans son discours de Vladivostok, Gorbachev disait encore: "L'U.R.S.S. est prête à examiner avec la Chine, à tout moment et à tout niveau et de la façon la plus sérieuse, des mesures visant à créer un climat de bon voisinage. Nous espérons que la frontière qui nous sépare - on aimerait dire : qui nous unit deviendra dans un proche avenir une zone de paix et d'amitié. Nos priorités et celles de la Chine sont similaires : accélérer le développement social et économique".

Sous le vernis de relations améliorées, la confrontation stratégique fondamentale entre les deux colosses asiatiques diminue-t-elle vraiment et l'activité soviétique dans le Pacifique aurait-elle été en fin de compte autre chose qu'une vaste manoeuvre destinée à encercler et à contenir une puissance chinoise dont le développement - idéologiquement hérétique doit demeurer un cauchemar pour une Russie hantée par le péril asiatique...?

La permanence d'un tel effort, dans les circonstances présentes, paraît toutefois dépasser largement les possibilités d'une U.R.S.S. acculée à la défensive, et Gorbachev a certainement pris la mesure du problème.

On s'oriente dès lors bien plus vers une collaboration pragmatique qui va en s'amplifiant : reprise d'une coopération industrielle et technologique, utilisation de main-d'oeuvre 
chinoise dans la Sibérie sous-peuplée, apaisement des litiges frontaliers sur le fleuve Amour.

En fin de compte, la Russie n'est-elle une puissance Pacifique que sur la carte ? Son histoire, ses ambitions, ses besoins ne sont-ils pas prioritairement orientés vers l'Europe ?

Il serait à coup sûr prématuré d'envisager un réel retrait soviétique de la zone Asie-Pacifique. Au contraire, ses intérêts économiques commandent à l'U.R.S.S. d'améliorer ses relations avec tous ses voisins extrême-orientaux. Vladimir Ivanov, président du département Pacifique de l'Institut des relations internationales de l'Académie, publiait dans le Far Eastern Economic Review du 22 février 1990 un article remarqué sous le titre "Perestroika in the Pacific". Il y souligne les grands progrès accomplis dans le rapprochement entre l'Union soviétique et la Corée du Sud, saluée comme un pont entre l'Asie orientale et l'Europe de l'Est. Et il espère que le Japon suivra une voie analogue. Mais, pour V. Ivanov, "l'esprit de la guerre froide" règne toujours dans le Pacifique Occidental. Il voit peu de signes de détente dans l'attitude des Etats-Unis, attachés à leur position dominante. Il rappelle que les manoeuvres navales "Pacex" menées conjointement par les Etats-Unis et le Japon constituaient "la plus vaste mobilisation dans l'histoire d'après-guerre de cette région". Mais l'appel du pied à un dialogue constructif, tant avec Washington qu'avec Tokyo - dans la foulée du discours de Gorbachev à Krasnoyarsk le 15 septembre 1988 montre que là aussi la situation reste mouvante et ouverte.

Quelles que soient les perspectives réalistes de voir la zone du Pacifique émerger comme "nouveau centre du monde", l'Europe doit s'y montrer attentive, relever les défis, oser y 
affirmer sa présence industrielle et commerciale. D'une manière générale, elle y sera la bienvenue, malgré les craintes loin d'être dissipées - qui s'expriment à l'égard de la création d'une "forteresse Europe" dont les marchés deviendraient moins accessibles.

La constitution de trois grands blocs plus ou moins antagonistes - Amérique du Nord, C.E.E., Asie Pacifique - n'est pas, en cas de recul du libre-échangisme au plan mondial, une perspective illusoire, malgré les dangers qu'elle recèle. A cet égard, il sera bon de garder à l'esprit le fait que les Etats-Unis occupent une position centrale, orientés, comme ils le sont, et parfois contradictoirement, vers l'Atlantique et vers le Pacifique. Il est permis de penser aussi que certains pays de l'Amérique latine, en premier lieu le Mexique, deviendront des partenaires plus actifs dans l'avenir dans cette zone. Des progrès se réaliseront dans la création, chère au gouvernement australien - discrètement encouragée par un Japon encore conscient des inquiétudes qu'il suscite, considérée avec prudence par les pays de l'ASEAN, - d'une "Communauté Economique de l'Asie Pacifique". Une double hégémonie nippo-américaine pourra y voir le jour.

Mais le rythme incroyablement accéléré des mutations de tout ordre que nous vivons depuis quelques mois doit inciter à la prudence dans les pronostics et inspirer à la Communauté Européenne une vigilance active, dans la pleine conscience du rôle qu'elle doit plus que jamais jouer dans ce nouvel ordre planétaire où le monde du Pacifique a pris une place de choix. 


\section{NOTES}

$1.44 \%$ des Asiato-Américains ont un diplôme universitaire. Pour l'ensemble de la population des Etats-Unis, ce chiffre est de $16 \%$.

2. Causerie à Bruxelles - 13/11/1984.

3. "Le Japon se sent peut-être moins que tout autre appartenir à l'Asie. Il a tendance à se penser un cas unique, culturellement à la périphérie $\mathrm{du}$ reste de la région, et d'abord comme un membre du camp occidental (Philippe Pons : le Japon comme grand ordinateur. Le Monde, 2 août 1988). 


\section{SUMMARY}

A broad description of the development prospects of the Pacific basin and the part that will probably be played by the principal states along its rim, i.e. the USA, Japan, the new industrialised economies in the Asia-Pacific region, Australia, China and the USSR.

The author calls upon Western Europe to strengthen its presence in the region and to ensure that freedom of trade is not abandoned to the advantage of large, inward- looking blocs. 